\title{
On the Interplay Between Channel Sensing and Estimation in Cognitive Radio Systems
}

\author{
Mustafa Cenk Gursoy \\ Department of Electrical Engineering \\ University of Nebraska-Lincoln, Lincoln, NE 68588 \\ Email: gursoy@engr.unl.edu
}

\author{
Sinan Gezici \\ Department of Electrical and Electronics Engineering \\ Bilkent University, Bilkent, Ankara 06800, Turkey \\ Email: gezici@ee.bilkent.edu.tr
}

\begin{abstract}
Cognitive radio transmissions in the presence of channel uncertainty are considered. In practical scenarios, cognitive secondary users need to perform both channel sensing in order to identify whether the channel is being occupied by the primary users or not, and also channel estimation in order to learn the channel fading coefficients. Generally, errors occur in both channel sensing and estimation, and this leads to a coupling between the two. More specifically, imperfect sensing affects both the structure and the performance of channel estimation schemes. With this motivation, the interactions between channel sensing and estimation are studied in this paper. In particular, different channel estimation schemes including minimum mean-square error (MMSE), linear MMSE, and mismatched MMSE estimations are analyzed, and their dependence on sensing decisions and their performances are investigated.

Index Terms-Cognitive radio, channel sensing, channel estimation, minimum mean-square error estimation.
\end{abstract}

\section{INTRODUCTION}

In cognitive radio networks, channel/spectrum sensing is one of the key tasks to be performed in order to limit the interference inflicted on the primary users. Due to the significance of this consideration, different spectrum sensing methods and dynamic spectrum access strategies have been extensively studied over the last couple of years (see e.g., [1]-[4]). Among frequently-used practical spectrum sensing methods such as matched filtering, energy detection, and cyclostationary detection, energy detection is commonly preferred if prior information about the structure of the primary users' signals is not available at the cognitive secondary users [4]. It is important to note that, as common to all schemes, errors in the form of false-alarms and miss-detections occur in sensing, and such errors can lead to degradation in the performance.

Another important concern in wireless systems is the estimation of the time-varying channel conditions which occur due to mobility and/or changing environment. In such cases, practical wireless systems generally employ training methods to estimate the channel fading coefficients, albeit imperfectly. On the other hand, despite their practical significance, channel estimation methods and communication in the presence of imperfectlyknown channel conditions have not been the focus of majority of the studies on cognitive radio systems. In [5], Gao et al. addressed channel training and estimation in a multiple-antenna cognitive radio setting. In their model, cognitive users initially listen to the primary users' transmission in order to learn the structure of the covariance matrices of the received signals and perform receive and transmit beamforming in their own transmissions. Following this phase, the cognitive users enter into a training phase in which pilot signals are sent and the linear minimum meansquare-error (LMMSE) estimation is performed. In a related study, an approach is proposed to perform spectrum sensing and data transmission simultaneously in order to maximize both the sensing time and the throughput of the cognitive system [6].

In this paper, we consider a practical setting in which cognitive secondary users operate under channel uncertainty, and we study the interactions between channel sensing and estimation. Initially, the secondary users sense the channel and make decisions on whether the channel is being occupied by the primary users or not. Subsequently, they perform channel estimation in order to learn the conditions in their own channels. The crucial assumption is that both channel sensing and estimation are performed with possible errors. Under this assumption, we show that imperfect channel sensing can have significant impact on the channel estimation, and both the structure of the estimators and the resulting average estimation errors have dependence on the sensing performance.

\section{Channel Sensing}

Initially, we assume that the secondary cognitive users sense the channel in order to identify whether the channel is being used by the primary users. Energy-detection methods are considered to be well-suited for channel sensing if the transmission policies of primary users are not known. In this case, we can formulate the channel sensing as a hypothesis testing problem between the noise $n_{i}$ and the signal $s_{i}$ in noise. If $N$ symbol periods are allocated for channel sensing, the hypothesis testing problem can mathematically be expressed as follows:

$$
\begin{aligned}
& \mathcal{H}_{0}: z_{i}=n_{i}, \quad i=1,2, \ldots, N, \\
& \mathcal{H}_{1}: z_{i}=s_{i}+n_{i}, \quad i=1,2, \ldots, N .
\end{aligned}
$$

Above, $s_{i}$ denotes the sum of the primary users' faded signals received by the cognitive user and is complex-valued. We assume that $s_{i}$ has a circularly-symmetric distribution with zeromean and variance $\sigma_{s}^{2}$. In addition, we assume that the signal samples $\left\{s_{i}\right\}$ are independent and identically distributed (i.i.d.). Furthermore, $\left\{n_{i}\right\}$ is a sequence of independent and identically distributed (i.i.d.), additive zero-mean, circularly symmetric, complex Gaussian noise samples with zero mean and variance $\mathbb{E}\left\{\left|n_{i}\right|^{2}\right\}=\sigma_{n}^{2}$ for all $i$. Under these assumptions, the optimal Neyman-Pearson detector for the above hypothesis problem is 
given by $Z=\frac{1}{N} \sum_{i=1}^{N}\left|z_{i}\right|^{2} \gtrless_{\mathcal{H}_{0}}^{\mathcal{H}_{1}} \lambda$ where $\lambda$ is the detection threshold.

Assuming that $N$ is sufficiently large, we can approximate $Z=\frac{1}{N} \sum_{i=1}^{N}\left|z_{i}\right|^{2}$ as a Gaussian random variable by invoking the Central Limit Theorem. It can easily be verified that the mean and variance of $Y$ are given, respectively, by [7]

$$
\begin{gathered}
\mathbb{E}\{Z\}=\left\{\begin{array}{ll}
\sigma_{n}^{2}, & \text { under } \mathcal{H}_{0} \\
\sigma_{s}^{2}+\sigma_{n}^{2}, & \text { under } \mathcal{H}_{1}
\end{array},\right. \text { and } \\
\operatorname{Var}\{Z\}= \begin{cases}\sigma_{n}^{4} / N, & \text { under } \mathcal{H}_{0} \\
\left(\mathbb{E}\left\{|s|^{4}\right\}+2 \sigma_{n}^{4}-\left(\sigma_{s}^{2}-\sigma_{n}^{2}\right)^{2}\right) / N, & \text { under } \mathcal{H}_{1}\end{cases}
\end{gathered}
$$

With these characterizations and the Gaussianity assumption, the detection and false-alarm probabilities can be expressed in terms of $Q$-functions [7]:

$$
\begin{aligned}
P_{d} & =\operatorname{Pr}\left\{Z>\lambda \mid \mathcal{H}_{1}\right\}=\operatorname{Pr}\left\{\hat{\mathcal{H}}_{1} \mid \mathcal{H}_{1}\right\} \\
& =Q\left(\frac{\lambda-\sigma_{s}^{2}-\sigma_{n}^{2}}{\sqrt{\frac{1}{N}\left(\mathbb{E}\left\{|s|^{4}\right\}+2 \sigma_{n}^{4}-\left(\sigma_{s}^{2}-\sigma_{n}^{2}\right)^{2}\right)}}\right) \text { and } \\
P_{f} & =\operatorname{Pr}\left\{Z>\lambda \mid \mathcal{H}_{0}\right\}=\operatorname{Pr}\left\{\hat{\mathcal{H}}_{1} \mid \mathcal{H}_{0}\right\}=Q\left(\frac{\lambda-\sigma_{n}^{2}}{\sqrt{\frac{1}{N} \sigma_{n}^{4}}}\right) .
\end{aligned}
$$

Above, $\hat{\mathcal{H}}_{1}$ is used to denote the event in which the cognitive users sense the channel as busy, i.e., declare $\mathcal{H}_{1}$ as the true hypothesis. Note that the true underlying hypothesis can be either $\mathcal{H}_{0}$ or $\mathcal{H}_{1}$. For instance, if $\mathcal{H}_{0}$ is actually the true hypothesis, $\operatorname{Pr}\left\{\hat{\mathcal{H}}_{1} \mid \mathcal{H}_{0}\right\}$ denotes the false alarm probability. Similarly, we denote by $\hat{\mathcal{H}}_{0}$ the event in which $\mathcal{H}_{0}$ is declared as the true hypothesis by the cognitive users. Hence, $\left\{\hat{\mathcal{H}}_{0}, \hat{\mathcal{H}}_{1}\right\}$ can equivalently be regarded as the possible channel sensing decisions.

In the above setting, $s_{i}$ is assumed to have an arbitrary, circularly-symmetric distribution with zero mean and finite variance $\sigma_{s}^{2}$. In the paper, besides treating the general scenarios, we also consider the special case in which $s_{i}$ has a Gaussian distribution. In such a case, we have $\mathbb{E}\left\{|s|^{4}\right\}=2 \sigma_{s}^{4}$ in (3). The Gaussian model for $s_{i}$ can be justified in cases in which the number of active primary users is large and hence $s_{i}$ is the sum of a large number of faded signals or in cases in which Rayleigh fading is experienced and the primary users employ phase or frequency modulation.

\section{Channel Estimation in the Presence of Sensing ERRORS}

Following the channel sensing phase, cognitive secondary users initiate the training phase in which the channel fading coefficient between the secondary transmitter and secondary receiver is estimated. We assume that the cognitive radio channel is subject to slow frequency-flat fading. To facilitate channel estimation, the secondary transmitter sends a pilot symbol. The pilot symbol power is assumed to depend on the sensing result. If the channel is detected as busy (i.e., sensing decision is $\hat{\mathcal{H}}_{1}$ ), the power of the pilot symbol is set to $P_{t 1}$. On the other hand, the pilot power is $P_{t 0}$ when no activity is detected and hence the sensing decision is $\hat{\mathcal{H}}_{0}$. The selection of two different power levels is again performed for the protection of the primary users. We assume that training in the presence of primary user activity is performed with smaller power and hence $P_{t 1}<P_{t 0}$. This limits the interference caused to the primary users. For instance, if no training and data transmission is performed when the channel is sensed as busy, then we can select $P_{t 1}=0$.

In this phase, we assume that the transmitter sends a single pilot symbol and the receiver observes its noisy version $y$ from which an estimate of the channel fading coefficient will be derived. Note that we have four possible scenarios and channel input-output relationships depending on the true channel states and the channel sensing decisions:

1) Scenario 1: Channel is busy, and is detected as busy, representing the joint event $\left(\mathcal{H}_{1}, \hat{\mathcal{H}}_{1}\right)$.

$$
y=h \sqrt{P_{t 1}}+n+s .
$$

2) Scenario 2: Channel is busy, but is detected as idle, representing the joint event $\left(\mathcal{H}_{1}, \hat{\mathcal{H}}_{0}\right)$.

$$
y=h \sqrt{P_{t 0}}+n+s .
$$

3) Scenario 3: Channel is idle, but is detected as busy, representing the joint event $\left(\mathcal{H}_{0}, \hat{\mathcal{H}}_{1}\right)$.

$$
y=h \sqrt{P_{t 1}}+n \text {. }
$$

4) Scenario 4: Channel is idle, and is detected as idle, representing the joint event $\left(\mathcal{H}_{0}, \hat{\mathcal{H}}_{0}\right)$.

$$
y=h \sqrt{P_{t 0}}+n .
$$

In the above channel input-output relationships, $h$ denotes the fading coefficient in the channel between the secondary transmitter and receiver, and it is assumed to be a zero-mean circularly-symmetric complex random variable with variance $\sigma_{h}^{2}$. As in the previous section, $n$ denotes the zero-mean complex Gaussian noise with variance $\sigma_{n}^{2}$, and $s$ is the sum of the faded primary users' signals arriving at the cognitive receiver.

Note that when the channel is busy, the additive disturbance is noise plus the primary users' received sum signal, i.e., $n+s$, as seen in (5) and (6), while only additive noise is present when the channel is not occupied by the primary users. Since errors are possible in channel sensing, the true state of the channel (busy or idle) and consequently the statistics of the additive disturbance are not perfectly known by the cognitive receiver. Hence, channel estimation needs to be performed in the presence of such sensing errors and ambiguities. Next, we analyze different estimation schemes.

\section{A. MMSE Estimation}

For a given channel sensing threshold $\lambda$, the MMSE estimate of the channel fading coefficient is obtained by solving

$$
\min _{\hat{h}} \mathbb{E}\left\{|h-\hat{h}|^{2}\right\}
$$

where $\hat{h}$ is any estimate that is a function of the observation $y$. Assume that the secondary receiver incorporates the channel sensing decision into channel estimation. Hence, the receiver obtains $\hat{h}_{0}$ under $\hat{\mathcal{H}}_{0}$ and $\hat{h}_{1}$ under $\hat{\mathcal{H}}_{1}$. Then, we can express 
the MMSE estimation problem as follows:

$$
\begin{aligned}
& \min _{\hat{h}_{0}, \hat{h}_{1}} \operatorname{Pr}\left\{\hat{\mathcal{H}}_{0}\right\} \mathbb{E}\left\{\left|h-\hat{h}_{0}\right|^{2} \mid \hat{\mathcal{H}}_{0}\right\}+\operatorname{Pr}\left\{\hat{\mathcal{H}}_{1}\right\} \mathbb{E}\left\{\left|h-\hat{h}_{1}\right|^{2} \mid \hat{\mathcal{H}}_{1}\right\} \\
= & \operatorname{Pr}\left\{\hat{\mathcal{H}}_{0}\right\} \min _{\hat{h}_{0}} \mathbb{E}\left\{\left|h-\hat{h}_{0}\right|^{2} \mid \hat{\mathcal{H}}_{0}\right\}+\operatorname{Pr}\left\{\hat{\mathcal{H}}_{1}\right\} \min _{\hat{h}_{1}} \mathbb{E}\left\{\left|h-\hat{h}_{1}\right|^{2} \mid \hat{\mathcal{H}}_{1}\right\}
\end{aligned}
$$

from which we see that the optimal MMSE estimates are given by

$$
\hat{h}_{m m s e, 0}=\mathbb{E}\left\{h \mid y, \hat{\mathcal{H}}_{0}\right\} \quad \text { and } \quad \hat{h}_{m m s e, 1}=\mathbb{E}\left\{h \mid y, \hat{\mathcal{H}}_{1}\right\} .
$$

The estimate $\hat{h}_{m m s e, 0}$ can further be expressed as

$$
\begin{aligned}
\hat{h}_{m m s e, 0}= & \mathbb{E}\left\{h \mid y, \hat{\mathcal{H}}_{0}\right\} \\
= & \operatorname{Pr}\left\{\mathcal{H}_{0} \mid \hat{\mathcal{H}}_{0}, y\right\} \mathbb{E}\left\{h \mid y, \mathcal{H}_{0}, \hat{\mathcal{H}}_{0}\right\} \\
& +\operatorname{Pr}\left\{\mathcal{H}_{1} \mid \hat{\mathcal{H}}_{0}, y\right\} \mathbb{E}\left\{h \mid y, \mathcal{H}_{1}, \hat{\mathcal{H}}_{0}\right\} .
\end{aligned}
$$

Using Bayes' rule, we can write

$\operatorname{Pr}\left\{\mathcal{H}_{0} \mid \hat{\mathcal{H}}_{0}, y\right\}=$

$$
\begin{aligned}
& \frac{\operatorname{Pr}\left\{\mathcal{H}_{0}\right\} \operatorname{Pr}\left\{\hat{\mathcal{H}}_{0} \mid \mathcal{H}_{0}\right\} f\left(y \mid \mathcal{H}_{0}, \hat{\mathcal{H}}_{0}\right)}{\operatorname{Pr}\left\{\mathcal{H}_{0}\right\} \operatorname{Pr}\left\{\hat{\mathcal{H}}_{0} \mid \mathcal{H}_{0}\right\} f\left(y \mid \mathcal{H}_{0}, \hat{\mathcal{H}}_{0}\right)+\operatorname{Pr}\left\{\mathcal{H}_{1}\right\} \operatorname{Pr}\left\{\hat{\mathcal{H}}_{0} \mid \mathcal{H}_{1}\right\} f\left(y \mid \mathcal{H}_{1}, \hat{\mathcal{H}}_{0}\right)} \\
& =\frac{\operatorname{Pr}\left\{\mathcal{H}_{0}\right\}\left(1-P_{f}\right) f\left(y \mid \mathcal{H}_{0}, \hat{\mathcal{H}}_{0}\right)}{\operatorname{Pr}\left\{\mathcal{H}_{0}\right\}\left(1-P_{f}\right) f\left(y \mid \mathcal{H}_{0}, \hat{\mathcal{H}}_{0}\right)+\operatorname{Pr}\left\{\mathcal{H}_{1}\right\}\left(1-P_{d}\right) f\left(y \mid \mathcal{H}_{1}, \hat{\mathcal{H}}_{0}\right)}
\end{aligned}
$$

where $f\left(y \mid \mathcal{H}_{0}, \hat{\mathcal{H}}_{0}\right)$ and $f\left(y \mid \mathcal{H}_{1}, \hat{\mathcal{H}}_{0}\right)$ denote the conditional distributions of the received signal $y$ given the true state of primary user activity and the sensing decision of the cognitive users, and $P_{f}$ and $P_{d}$ denote the false alarm and detection probabilities, respectively. Moreover, $\operatorname{Pr}\left\{\mathcal{H}_{0}\right\}$ and $\operatorname{Pr}\left\{\mathcal{H}_{1}\right\}$ are the prior probabilities of channel being idle and busy, respectively. Note also that

$$
\operatorname{Pr}\left\{\mathcal{H}_{1} \mid \hat{\mathcal{H}}_{0}, y\right\}=1-\operatorname{Pr}\left\{\mathcal{H}_{0} \mid \hat{\mathcal{H}}_{0}, y\right\}
$$

Similarly, we can express the estimate $\hat{h}_{m m s e, 1}$ as

$$
\begin{aligned}
\hat{h}_{m m s e, 1}= & \mathbb{E}\left\{h \mid y, \hat{\mathcal{H}}_{1}\right\} \\
= & \operatorname{Pr}\left\{\mathcal{H}_{0} \mid \hat{\mathcal{H}}_{1}, y\right\} \mathbb{E}\left\{h \mid y, \mathcal{H}_{0}, \hat{\mathcal{H}}_{1}\right\} \\
& +\operatorname{Pr}\left\{\mathcal{H}_{1} \mid \hat{\mathcal{H}}_{1}, y\right\} \mathbb{E}\left\{h \mid y, \mathcal{H}_{1}, \hat{\mathcal{H}}_{1}\right\}
\end{aligned}
$$

where

$\operatorname{Pr}\left\{\mathcal{H}_{0} \mid \hat{\mathcal{H}}_{1}, y\right\}=$

$\frac{\operatorname{Pr}\left\{\mathcal{H}_{0}\right\} \operatorname{Pr}\left\{\hat{\mathcal{H}}_{1} \mid \mathcal{H}_{0}\right\} f\left(y \mid \mathcal{H}_{0}, \hat{\mathcal{H}}_{1}\right)}{\operatorname{Pr}\left\{\mathcal{H}_{0}\right\} \operatorname{Pr}\left\{\hat{\mathcal{H}}_{1} \mid \mathcal{H}_{0}\right\} f\left(y \mid \mathcal{H}_{0}, \hat{\mathcal{H}}_{1}\right)+\operatorname{Pr}\left\{\mathcal{H}_{1}\right\} \operatorname{Pr}\left\{\hat{\mathcal{H}}_{1} \mid \mathcal{H}_{1}\right\} f\left(y \mid \mathcal{H}_{1}, \hat{\mathcal{H}}_{1}\right)}$

$$
=\frac{\operatorname{Pr}\left\{\mathcal{H}_{0}\right\} P_{f} f\left(y \mid \mathcal{H}_{0}, \hat{\mathcal{H}}_{0}\right)}{\operatorname{Pr}\left\{\mathcal{H}_{0}\right\} P_{f} f\left(y \mid \mathcal{H}_{0}, \hat{\mathcal{H}}_{1}\right)+\operatorname{Pr}\left\{\mathcal{H}_{1}\right\} P_{d} f\left(y \mid \mathcal{H}_{1}, \hat{\mathcal{H}}_{1}\right)}
$$

and

$$
\operatorname{Pr}\left\{\mathcal{H}_{1} \mid \hat{\mathcal{H}}_{1}, y\right\}=1-\operatorname{Pr}\left\{\mathcal{H}_{0} \mid \hat{\mathcal{H}}_{1}, y\right\}
$$

Remark 1: As seen above, channel sensing errors have an impact on the MMSE estimates through the false alarm and missdetection probabilities, $P_{f}$ and $\left(1-P_{d}\right)$. In particular, we note that the conditional probabilities $\operatorname{Pr}\left\{\mathcal{H}_{i} \mid \hat{\mathcal{H}}_{j}, y\right\}$ for $i, j \in\{0,1\}$ are rather complicated involving the conditional probability density functions $f\left(y \mid \mathcal{H}_{i}, \hat{\mathcal{H}}_{j}\right)$. When channel sensing is perfect and consequently $P_{d}=1$ and $P_{f}=0$, then we have

$$
\operatorname{Pr}\left\{\mathcal{H}_{i} \mid \hat{\mathcal{H}}_{j}, y\right\}=\left\{\begin{array}{ll}
1 & \text { if } i=j \\
0 & \text { if } i \neq j
\end{array},\right.
$$

leading to significant simplifications in the estimate expressions.

1) MMSE Estimation in the Gaussian Model: Above, we have considered a general scenario in which the primary users' total received signal $s$ and the fading coefficient $h$ have arbitrary distributions with zero-mean and finite variances. In this subsection, we address a special case where $s$ and $h$ are independent, circularly-symmetric Gaussian random variables. Note that $y$ is now Gaussian distributed as well, and we can express the MMSE estimates as follows:

$$
\begin{aligned}
\hat{h}_{m m s e, 0}= & \operatorname{Pr}\left\{\mathcal{H}_{0} \mid \hat{\mathcal{H}}_{0}, y\right\} \frac{\sqrt{P_{t 0}} \sigma_{h}^{2}}{P_{t 0} \sigma_{h}^{2}+\sigma_{n}^{2}} y \\
& +\operatorname{Pr}\left\{\mathcal{H}_{1} \mid \hat{\mathcal{H}}_{0}, y\right\} \frac{\sqrt{P_{t 0}} \sigma_{h}^{2}}{P_{t 0} \sigma_{h}^{2}+\sigma_{n}^{2}+\sigma_{s}^{2}} y, \quad \text { and } \\
\hat{h}_{m m s e, 1}= & \operatorname{Pr}\left\{\mathcal{H}_{0} \mid \hat{\mathcal{H}}_{1}, y\right\} \frac{\sqrt{P_{t 1}} \sigma_{h}^{2}}{P_{t 1} \sigma_{h}^{2}+\sigma_{n}^{2}} y \\
& +\operatorname{Pr}\left\{\mathcal{H}_{1} \mid \hat{\mathcal{H}}_{1}, y\right\} \frac{\sqrt{P_{t 1}} \sigma_{h}^{2}}{P_{t 1} \sigma_{h}^{2}+\sigma_{n}^{2}+\sigma_{s}^{2}} y .
\end{aligned}
$$

These MMSE estimates are determined by using the fact that the conditional expectations for Gaussian random variables are given by

$$
\mathbb{E}\left\{h \mid y, \mathcal{H}_{i}, \hat{\mathcal{H}}_{j}\right\}=\frac{\mathbb{E}\left\{h y^{*} \mid \mathcal{H}_{i}, \hat{\mathcal{H}}_{j}\right\}}{\mathbb{E}\left\{|y|^{2} \mid \mathcal{H}_{i}, \hat{\mathcal{H}}_{j}\right\}} y
$$

Note that once the joint event $\left(\mathcal{H}_{i}, \hat{\mathcal{H}}_{j}\right)$ is given, we can determine the scenario and the corresponding the input-output relationship from the ones given in (5)-(8), and easily evaluate the expectations on the right-hand side of (23).

Additionally, in a Gaussian setting, the conditional probability density function of $y$, which we have in the conditional probability expressions in (13) and (18), becomes

$$
f\left(y \mid \mathcal{H}_{i}, \hat{\mathcal{H}}_{j}\right)=\frac{1}{\pi\left(P_{t j} \sigma_{h}^{2}+\sigma_{i}^{2}\right)} \mathrm{e}^{-\frac{|y|^{2}}{P_{t j} \sigma_{h}^{2}+\sigma_{i}^{2}}} \text { for } i, j \in\{0,1\}
$$

where $\sigma_{i}^{2}=\left\{\begin{array}{ll}\sigma_{n}^{2}, & \text { if } i=0 \\ \sigma_{n}^{2}+\sigma_{s}^{2}, & \text { if } i=1\end{array}\right.$.

\section{B. Linear MMSE Estimation}

We experience two difficulties in the analysis of MMSE estimation. First, it is difficult to come up with closed-form expressions for the estimates for arbitrary distributions of $s$ and $h$. Secondly, even in the Gaussian setting in which the estimates can be obtained in closed-form, computing the mean-square errors seems intractable due to the presence of conditional distributions of $y$ in both the numerator and the denominator of (13) and (18). We can determine the mean-square errors through simulations.

These issues can be alleviated by considering suboptimal strategies. In this subsection, we study linear MMSE estimation. Note that given the observation $y$, the linear MMSE estimate under sensing decision $\hat{\mathcal{H}}_{0}$ (i.e., when the channel is sensed as 
idle) is

$$
\begin{aligned}
& \hat{h}_{\text {lmmse }, 0}=\frac{\mathbb{E}\left\{h y^{*} \mid \hat{\mathcal{H}}_{0}\right\}}{\mathbb{E}\left\{|y|^{2} \mid \hat{\mathcal{H}}_{0}\right\}} y=\frac{\sqrt{P_{t 0}} \sigma_{h}^{2}}{\mathbb{E}\left\{|y|^{2} \mid \hat{\mathcal{H}}_{0}\right\}} y \\
& =\frac{\sqrt{P_{t 0}} \sigma_{h}^{2}}{\operatorname{Pr}\left\{\mathcal{H}_{0} \mid \hat{\mathcal{H}}_{0}\right\} \mathbb{E}\left\{|y|^{2} \mid \mathcal{H}_{0}, \hat{\mathcal{H}}_{0}\right\}+\operatorname{Pr}\left\{\mathcal{H}_{1} \mid \hat{\mathcal{H}}_{0}\right\} \mathbb{E}\left\{|y|^{2} \mid \mathcal{H}_{1}, \hat{\mathcal{H}}_{0}\right\}} y \\
& =\frac{\sqrt{P_{t 0}} \sigma_{h}^{2}}{\operatorname{Pr}\left\{\mathcal{H}_{0} \mid \hat{\mathcal{H}}_{0}\right\}\left(P_{t 0} \sigma_{h}^{2}+\sigma_{n}^{2}\right)+\operatorname{Pr}\left\{\mathcal{H}_{1} \mid \hat{\mathcal{H}}_{0}\right\}\left(P_{t 0} \sigma_{h}^{2}+\sigma_{n}^{2}+\sigma_{s}^{2}\right)} y
\end{aligned}
$$

$=a_{0} y$

where $a_{0}$ is the constant representing the fraction in (26). Similarly, the linear MMSE estimate when the channel is detected as busy is

$$
\begin{aligned}
& \hat{h}_{\text {lmmse,1 }}=\frac{\mathbb{E}\left\{h y^{*} \mid \hat{\mathcal{H}}_{1}\right\}}{\mathbb{E}\left\{|y|^{2} \mid \hat{\mathcal{H}}_{1}\right\}} y=\frac{\sqrt{P_{t 1}} \sigma_{h}^{2}}{\mathbb{E}\left\{|y|^{2} \mid \hat{\mathcal{H}}_{1}\right\}} y \\
& =\frac{\sqrt{P_{t 1}} \sigma_{h}^{2}}{\operatorname{Pr}\left\{\mathcal{H}_{0} \mid \hat{\mathcal{H}}_{1}\right\} \mathbb{E}\left\{|y|^{2} \mid \mathcal{H}_{0}, \hat{\mathcal{H}}_{1}\right\}+\operatorname{Pr}\left\{\mathcal{H}_{1} \mid \hat{\mathcal{H}}_{1}\right\} \mathbb{E}\left\{|y|^{2} \mid \mathcal{H}_{1}, \hat{\mathcal{H}}_{1}\right\}} y \\
& =\frac{\sqrt{P_{t 1}} \sigma_{h}^{2}}{\operatorname{Pr}\left\{\mathcal{H}_{0} \mid \hat{\mathcal{H}}_{1}\right\}\left(P_{t 1} \sigma_{h}^{2}+\sigma_{n}^{2}\right)+\operatorname{Pr}\left\{\mathcal{H}_{1} \mid \hat{\mathcal{H}}_{1}\right\}\left(P_{t 1} \sigma_{h}^{2}+\sigma_{n}^{2}+\sigma_{s}^{2}\right)} y \\
& =a_{1} y
\end{aligned}
$$

Remark 2: The linear MMSE expressions in (27) and (29) depend on $s$ and $h$ only through their variances $\sigma_{s}^{2}$ and $\sigma_{h}^{2}$, and hence apply to different distributions with the same first (zeromean) and second-order statistics.

Remark 3: It is also interesting to note that even in the Gaussian setting, linear MMSE estimates and the MMSE estimates given in (21) and (22) are not equal due to sensing errors and the resulting uncertainty in the additive disturbance (see Section IV). If sensing is perfect (i.e., $P_{d}=1$ and $P_{f}=0$ ), these estimates become the same as expected.

The mean-square error (MSE) of linear MMSE estimation can be computed from

$$
\begin{aligned}
\mathrm{MSE}= & \operatorname{Pr}\left\{\hat{\mathcal{H}}_{0}\right\} \mathbb{E}\left\{\left|h-\hat{h}_{\text {lmmse }, 0}\right|^{2} \mid \hat{\mathcal{H}}_{0}\right\} \\
& +\operatorname{Pr}\left\{\hat{\mathcal{H}}_{1}\right\} \mathbb{E}\left\{\left|h-\hat{h}_{\text {lmmse, }}\right|^{2} \mid \hat{\mathcal{H}}_{1}\right\} \\
= & \operatorname{Pr}\left\{\mathcal{H}_{0}\right\} \operatorname{Pr}\left\{\hat{\mathcal{H}}_{0} \mid \mathcal{H}_{0}\right\} \mathbb{E}\left\{\left|h-\hat{h}_{\text {lmmse }, 0}\right|^{2} \mid \mathcal{H}_{0}, \hat{\mathcal{H}}_{0}\right\} \\
& +\operatorname{Pr}\left\{\mathcal{H}_{1}\right\} \operatorname{Pr}\left\{\hat{\mathcal{H}}_{0} \mid \mathcal{H}_{1}\right\} \mathbb{E}\left\{\left|h-\hat{h}_{\text {lmmse }, 0}\right|^{2} \mid \mathcal{H}_{1}, \hat{\mathcal{H}}_{0}\right\} \\
& +\operatorname{Pr}\left\{\mathcal{H}_{0}\right\} \operatorname{Pr}\left\{\hat{\mathcal{H}}_{1} \mid \mathcal{H}_{0}\right\} \mathbb{E}\left\{\left|h-\hat{h}_{\text {lmmse }, 1}\right|^{2} \mid \mathcal{H}_{0}, \hat{\mathcal{H}}_{1}\right\} \\
& +\operatorname{Pr}\left\{\mathcal{H}_{1}\right\} \operatorname{Pr}\left\{\hat{\mathcal{H}}_{1} \mid \mathcal{H}_{1}\right\} \mathbb{E}\left\{\left|h-\hat{h}_{\text {lmmse, }}\right|^{2} \mid \mathcal{H}_{1}, \hat{\mathcal{H}}_{1}\right\} \\
= & \operatorname{Pr}\left\{\mathcal{H}_{0}\right\}\left(1-P_{f}\right) \mathbb{E}\left\{\left|h-\hat{h}_{l m m s e, 0}\right|^{2} \mid \mathcal{H}_{0}, \hat{\mathcal{H}}_{0}\right\} \\
& +\operatorname{Pr}\left\{\mathcal{H}_{1}\right\}\left(1-P_{d}\right) \mathbb{E}\left\{\left|h-\hat{h}_{\text {lmmse, }}\right|^{2} \mid \mathcal{H}_{1}, \hat{\mathcal{H}}_{0}\right\} \\
& +\operatorname{Pr}\left\{\mathcal{H}_{0}\right\} P_{f} \mathbb{E}\left\{\left|h-\hat{h}_{l m m s e, 1}\right|^{2} \mid \mathcal{H}_{0}, \hat{\mathcal{H}}_{1}\right\} \\
& +\operatorname{Pr}\left\{\mathcal{H}_{1}\right\} P_{d} \mathbb{E}\left\{\left|h-\hat{h}_{l m m s e, 1}\right|^{2} \mid \mathcal{H}_{1}, \hat{\mathcal{H}}_{1}\right\}
\end{aligned}
$$

In the MSE expression above, we can easily obtain the conditional error variances as

$$
\begin{aligned}
\mathbb{E}\left\{\left|h-\hat{h}_{l m m s e, j}\right|^{2} \mid \mathcal{H}_{i}, \hat{\mathcal{H}}_{j}\right\}= & \sigma_{\hat{h}_{l m m s e, j} \mid \mathcal{H}_{i}, \hat{\mathcal{H}}_{j}}^{2} \\
& +\left(1-2 a_{j} \sqrt{P_{t j}}\right) \sigma_{h}^{2}
\end{aligned}
$$

where the conditional variance of the linear MMSE estimate is

$$
\begin{aligned}
\sigma_{\hat{h}_{l m m s e, j} \mid \mathcal{H}_{i}, \hat{\mathcal{H}}_{j}}^{2} & =\mathbb{E}\left\{\left|\hat{h}_{\text {lmmse }, j}\right|^{2} \mid \mathcal{H}_{i}, \hat{\mathcal{H}}_{j}\right\} \\
& =\left\{\begin{array}{ll}
a_{j}^{2}\left(P_{t j} \sigma_{h}^{2}+\sigma_{n}^{2}\right), & \text { if } i=0 \\
a_{j}^{2}\left(P_{t j} \sigma_{h}^{2}+\sigma_{n}^{2}+\sigma_{s}^{2}\right), & \text { if } i=1
\end{array},\right.
\end{aligned}
$$

and $a_{j}$ for $j \in\{0,1\}$ is the scaling constant in the linear MMSE estimates (27) and (29).

\section{Mismatched MMSE Estimation}

In this scheme, the receiver estimates the fading coefficients assuming a Gaussian setting and regarding the channel sensing result as perfect with no errors. Under these assumptions, the channel estimates under $\hat{\mathcal{H}}_{0}$ and $\hat{\mathcal{H}}_{1}$ are, respectively,

$$
\begin{aligned}
& \hat{h}_{m-m m s e, 0}=\frac{\sqrt{P_{t 0}} \sigma_{h}^{2}}{P_{t 0} \sigma_{h}^{2}+\sigma_{n}^{2}} y=b_{0} y \text { and } \\
& \hat{h}_{m-m m s e, 1}=\frac{\sqrt{P_{t 1}} \sigma_{h}^{2}}{P_{t 1} \sigma_{h}^{2}+\sigma_{n}^{2}+\sigma_{s}^{2}} y=b_{1} y .
\end{aligned}
$$

Note that the estimates above are the MMSE estimates in the case in which the primary users' total faded signal $s$ and the fading coefficient $h$ are Gaussian distributed, and channel sensing is performed with no errors (i.e., $P_{d}=1$ and $P_{f}=0$ ). In the absence of these assumptions, there is a mismatch in the model and these estimates are clearly suboptimal. Due to these, we call these estimates as mismatched MMSE (m-MMSE) estimates.

The MSE for m-MMSE estimates can be found using the same formulation as in (32) with

$$
\begin{aligned}
\mathbb{E}\left\{\left|h-\hat{h}_{m-m m s e, j}\right|^{2} \mid \mathcal{H}_{i}, \hat{\mathcal{H}}_{j}\right\}= & \sigma_{\hat{h}_{l m m s e, j} \mid \mathcal{H}_{i}, \hat{\mathcal{H}}_{j}}^{2} \\
& +\left(1-2 b_{j} \sqrt{P_{t j}}\right) \sigma_{h}^{2}
\end{aligned}
$$

and

$$
\begin{aligned}
\sigma_{\hat{h}_{m-m m s e, j} \mid \mathcal{H}_{i}, \hat{\mathcal{H}}_{j}}^{2} & =\mathbb{E}\left\{\left|\hat{h}_{m-m m s e, j}\right|^{2} \mid \mathcal{H}_{i}, \hat{\mathcal{H}}_{j}\right\} \\
& = \begin{cases}b_{j}^{2}\left(P_{t j} \sigma_{h}^{2}+\sigma_{n}^{2}\right), & \text { if } i=0 \\
b_{j}^{2}\left(P_{t j} \sigma_{h}^{2}+\sigma_{n}^{2}+\sigma_{s}^{2}\right), & \text { if } i=1\end{cases}
\end{aligned}
$$

for $j \in\{0,1\}$.

\section{Simulation Results}

In this section, simulation results are presented in order to compare various channel estimation algorithms in the presence of channel sensing errors. It is assumed that primary users are present in the environment with a probability of 0.25 ; that is, $\operatorname{Pr}\left\{\mathcal{H}_{1}\right\}=0.25$ and $\operatorname{Pr}\left\{\mathcal{H}_{0}\right\}=0.75$. In the absence of primary users, the cognitive user sets the power of its pilot symbol to $P_{t 0}=1$, whereas it reduces this power by $10 \mathrm{~dB}$ in the presence of primary users; i.e., $P_{t 1}=0.1$. Also, the fading coefficient $h$ in (5)-(8) is modeled as a circularly symmetric, complex Gaussian random variable with zero mean and $\mathbb{E}\left\{|h|^{2}\right\}=\sigma_{h}^{2}=1$. Similarly, the sum of the faded primary users' signals $s$ in (5) and (6) is a circularly symmetric, complex Gaussian random variable with zero mean and $\sigma_{s}^{2}=1$.

In Fig. 1, the mean-square errors (MSEs) of the MMSE estimator, the linear MMSE estimator, and the mismatched MMSE estimator are plotted versus the noise variance, $\sigma_{n}^{2}$. The sensing unit is modeled to have a detection probability of 


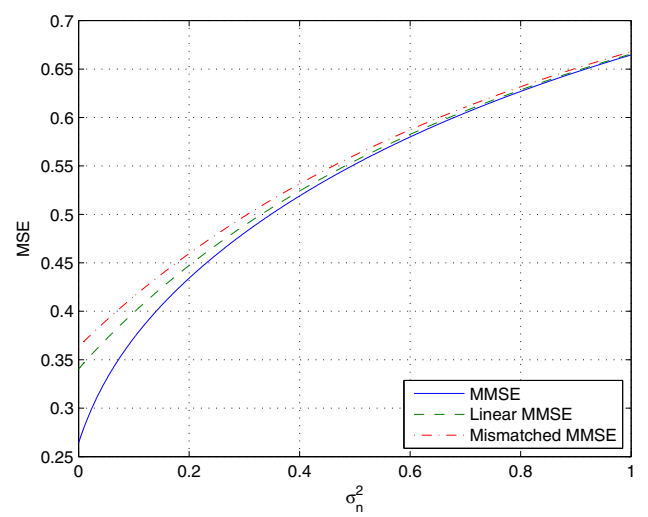

Fig. 1. MSE vs. average noise power for the MMSE estimator, the linear MMSE estimator. and the mismatched MMSE estimator.

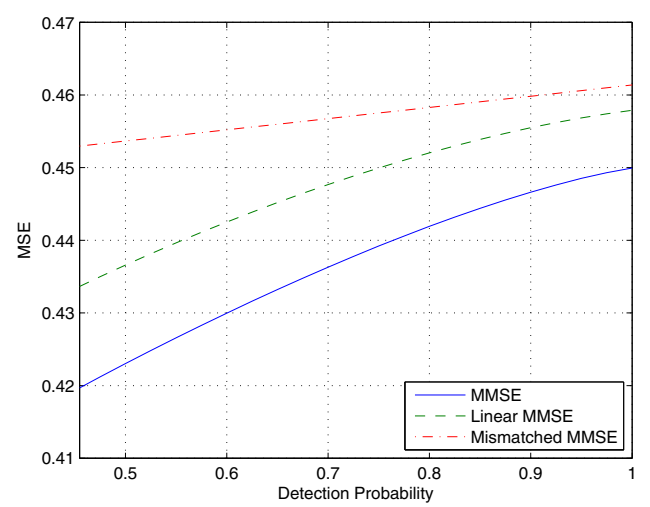

Fig. 2. MSE vs. detection probability for the MMSE estimator, the linear MMSE estimator, and the mismatched MMSE estimator.

$P_{d}=0.6$ and a false-alarm probability of $P_{f}=0.2$. It is observed that the MMSE estimator achieves the lowest MSEs whereas the mismatched MMSE has the worst performance, as expected. In addition, as the noise variance increases, the MSEs increase and the performance of the estimators get close to each other. Another important observation is that although Gaussian fading coefficients and signals are considered, the linear MMSE estimator is different from the MMSE estimator (it is well-known that the MMSE estimator reduces to the linear MMSE estimator when the observations are Gaussian distributed). This is mainly due to the fact that the observations come from four different scenarios as in (5)-(8) depending on the true hypothesis and the channel sensing decision; hence, the observations are in fact Gaussian mixture random variables.

In order to investigate the performance of the estimators for various detection probabilities, a scenario with a fixed false-alarm probability $\left(P_{f}=0.2\right)$ and a fixed noise variance $\left(\sigma_{n}^{2}=0.2\right)$ is considered, and Fig. 2 is obtained. ${ }^{1}$ As in the previous case, the MMSE estimator has the best performance and the mismatched MMSE estimator has the highest MSEs. Also, the MSEs increase as the detection probability increases. This seemingly counterintuitive result is due to the fact that whenever primary users are detected, the cognitive user needs to reduce the power of its pilot symbol by $10 \mathrm{~dB}\left(P_{t 1}=0.1\right.$ is used instead of $\left.P_{t 0}=1\right)$; hence, the channel estimation errors increase. In other words, lower MSEs can be obtained as the detection probability is reduced.

\footnotetext{
${ }^{1}$ It should be noted that the MSEs are plotted in Fig. 2 for detection probabilities above 0.4547 since lower detection probabilities cannot be obtained for $P_{f}=0.2$ and $\sigma_{n}^{2}=0.2$ according to (3) and (4).
}

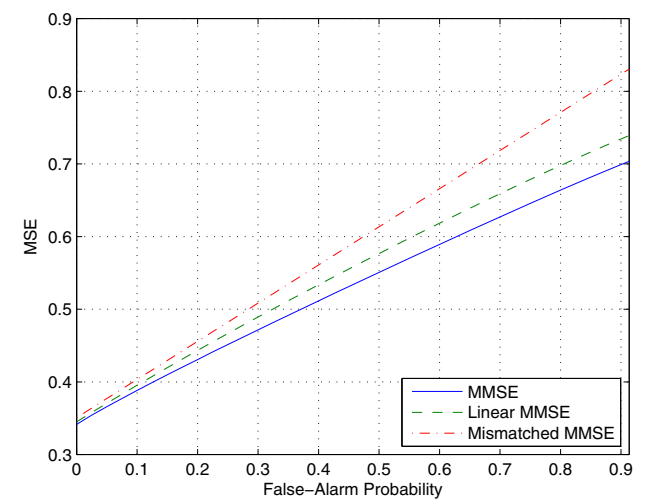

Fig. 3. MSE vs. false-alarm probability for the MMSE estimator, the linear MMSE estimator, and the mismatched MMSE estimator.

However, it should be noted that the detection probability cannot be smaller than a certain threshold in order to constrain the amount of interference from the cognitive user to the primary users.

Finally, in Fig. 3, the MSEs of the MMSE estimator, the linear MMSE estimator, and the mismatched MMSE estimator are plotted versus the false-alarm probability for a detection probability of $P_{d}=0.6$ and a noise variance of $\sigma_{n}^{2}=0.2$. It is observed that the MSEs increase as the false-alarm probability increases. This is mainly because the power of the pilot symbol is reduced ( $P_{t 1}=0.1$ is employed) in the presence of a false alarm; that is, when the channel sensing unit decides that the primary users are present in the system when in fact they are not. Also, as expected, the MMSE estimator achieves the best performance for false-alarm probabilities.

\section{CONCLUding REMARKS}

Channel estimation has been studied for cognitive radio systems in the presence of channel sensing errors, and the interplay between channel sensing and estimation has been investigated. An MMSE estimator that takes scenarios corresponding to possible channel sensing decisions and true channel states into account has been proposed. In addition, a simplified approach that employs linear MMSE estimator has been considered. The performance of these estimators have been evaluated and compared to the mismatched MMSE estimator, which assumes that the channel sensing decisions are perfect. The performance improvements have been observed in terms of reduced MSEs.

\section{REFERENCES}

[1] Q. Zhao and B. Sadler, "A survey of dynamic spectrum access," IEEE Sig. Pro. Mag., vol. 24, no. 3, pp. 79-89, May 2007.

[2] A. Ghasemi and E. S. Sousa, "Spectrum sensing in cognitive radio networks: Requirements, challenges and design trade-offs," IEEE Comm. Mag., vol. 46, no, 4, pp. 32-39, Apr. 2008.

[3] H. Arslan, Cognitive Radio, Software Defined Radio, and Adaptive Wireless Systems. New York: Springer, 2007.

[4] E. Hossain, D. Niyato, and Z. Han, Dynamic Spectrum Access and Management in Cognitive Radio Networks, Cambridge University Press, 2009.

[5] F. Gao, R. Zhang, Y.-C. Liang, and X. Wang, "Design of learning-based MIMO cognitive radio systems," IEEE Trans. Vehicular Technology, vol. 59, no. 4., pp. 1707-1720, May 2010.

[6] S. Stotas and A. Nallanathan, "Overcoming the sensing-throughput tradeoff in cognitive radio networks," Proc. IEEE Int. Conf. on Commun., Cape Town, May 2010.

[7] Y.-C. Liang, Y. Zheng, E. C. Y. Peh, and A. T. Hoang, "Sensing-throughput tradeoff for cognitive radio networks," IEEE Trans. Wireless Commun., vol. 7, no. 4, pp. 1326-1337, Apr. 2008. 